\title{
Genome-wide mapping of promoter-anchored interactions with close to single-enhancer resolution
}

\author{
Pelin Sahlén ${ }^{1 *}$, Ilgar Abdullayev²,3†, Daniel Ramsköld ${ }^{2,3}$, Liudmila Matskova $^{3}$, Nemanja Rilakovic ${ }^{1}$, Britta Lötstedt ${ }^{1}$, \\ Thomas J. Albert ${ }^{4}$, Joakim Lundeberg ${ }^{1}$ and Rickard Sandberg ${ }^{2,3^{*}}$ (i)
}

\begin{abstract}
Although the locations of promoters and enhancers have been identified in several cell types, we still have limited information on their connectivity. We developed HiCap, which combines a 4-cutter restriction enzyme $\mathrm{Hi}-\mathrm{C}$ with sequence capture of promoter regions. Applying the method to mouse embryonic stem cells, we identified promoter-anchored interactions involving 15,905 promoters and 71,984 distal regions. The distal regions were enriched for enhancer marks and transcription, and had a mean fragment size of only $699 \mathrm{bp}$ - close to single-enhancer resolution. High-resolution maps of promoter-anchored interactions with HiCap will be important for detailed characterizations of chromatin interaction landscapes.
\end{abstract}

\section{Background}

Enhancers are cis-acting DNA elements, essential for the regulation of transcription at nearby genes [1]. Although numerous methods exist for the genome-wide mapping of enhancers, e.g., STARR-seq [2] and ChIP-seq for transcription factors (TFs) [3], co-factors [4], chromatin modifications [5], and DNA hypersensitive sites [6], it is still challenging to globally identify the promoters regulated by each enhancer. Since enhancer regulation is mediated via genome looping, which physically brings distant regions into close proximity [7], selected promoter-enhancer interactions can be investigated using chromatin conformation capture (3C) [8]. Using a specific region as bait (e.g., a promoter), chromosome conformation capture coupled with sequencing $(4 \mathrm{C})[9,10]$ can be used to map genomewide interactions with the bait region at high sensitivity and resolution. Genome-wide chromatin interaction was first studied de novo with the development of $\mathrm{Hi}-\mathrm{C}$ [11] that selected for ligated fragments without using any particular regions as baits. This method was successfully used to identify topological domains and higher-order

\footnotetext{
* Correspondence: pelin.akan@scilifelab.se; rickard.sandberg@ki.se ${ }^{\dagger}$ Equal contributors

'KTH - Royal Institute of Technology, Science for Life Laboratory, School of Biotechnology, Solna 171 65, Sweden

${ }^{2}$ Ludwig Institute for Cancer Research, Stockholm 171 77, Sweden Full list of author information is available at the end of the article
}

chromatin interaction patterns [12]; however, its 5-20 kb resolution prevents mapping of individual promoter-enhancer interactions [13], and improvement in resolution scales with the square of the sequence depth. Chromatin interaction analysis by paired-end tag sequencing (ChIAPET) was developed to enrich for long-range interactions involving specific DNA binding factors [14] or actively transcribed regions [15]. Although ChIA-PET has higher resolution than $\mathrm{Hi}-\mathrm{C}$, the dependence on specific proteins for the immunoprecipitation reduces analyses to specific enhancers or actively transcribed genes. In parallel to the development of ChIA-PET, capture probes have been designed to hundreds of specific chromatin regions to improve $3 \mathrm{C}$ resolution, in a method called Capture-C [16]. Recently, genome-wide interaction maps have been generated by combining $\mathrm{Hi}-\mathrm{C}$ with capture probes targeting all promoters. They employed six-cutter restriction enzymes and obtained detailed chromatin maps at an average resolution of $3.4 \mathrm{~kb}[17,18]$. Another recent study [19] combined Hi-C with capture probes against 998 long non-coding RNA genes. Using DNase I instead of a restriction enzyme, they obtained smaller fragment size and the identification of hundreds of interactions at $1 \mathrm{~kb}$ resolution.

In this study we have developed HiCap, enabling the generation of genome-wide maps of promoter-anchored 
chromatin interactions with close to single-enhancer resolution. A strong enrichment was observed for interactions with distal regions harboring enhancer-associated marks and those were frequently transcribed. Additionally, we demonstrate that HiCap interactions contain gene regulatory information through integrative analyses of TF overexpression and genome-wide binding (ChIP-seq) data.

\section{Results}

\section{Development of HiCap}

To identify genome-wide interactions anchored on promoters, we started by experimenting with $3 \mathrm{C}$ and $\mathrm{Hi}-\mathrm{C}$ procedures together with sequence capture of promoter regions. We constructed capture probes that targeted restriction fragments containing the annotated promoters for essentially all mouse genes $(31,127$ promoters in 16,696 unique genes) and additional control regions in intergenic regions and exons $(n=184)$ (Table S1 in Additional file 1). We first investigated extensions of the Capture-C procedure to genome-wide level (by coupling $3 \mathrm{C}$ with sequence capture), but observed that Capture-C strongly enriched for un-ligated fragments, producing few read pairs with informative (>1 kb apart) junctions (Fig. S1 in Additional file 2). Instead, we based HiCap on modified $\mathrm{Hi}-\mathrm{C}$ followed by a sequence capture of promotercontaining fragments (Fig. S2 in Additional file 2). While published Capture-C libraries [16] contain 1.3-2.5\% read pairs with informative connectivity information (i.e., a $1 \mathrm{~kb}$ to $10 \mathrm{Mb}$ distance between the read pair), the HiCap libraries had much higher content (26-46 \%) of such read pairs (Fig. S1 in Additional file 2). We calculated library complexity, i.e., the number of unique DNA fragments, using Preseq [20], which extrapolates from read duplicate frequency, and found that the HiCap libraries also had higher complexity than Capture- $C$ libraries per input amounts of cells (7.7-fold difference, $P=0.009$, $t$-test; Table S2 in Additional file 1). To obtain high-resolution interactions, we performed the $\mathrm{Hi}-\mathrm{C}$ step of $\mathrm{HiCap}$ using a 4-cutter (MboI), which has a theoretical mean fragment size of only $422 \mathrm{bp}$ in the mouse genome (Fig. S3 in Additional file 2). We generated two HiCap libraries (biological replicates) from mouse embryonic stem cells (mESCs) and sequenced the libraries from both ends $(2 \times 100 \mathrm{bp})$ to a depth of 200-300 million read pairs. HiCap reads were mapped independently and read pairs were discarded if they mapped within $1 \mathrm{~kb}$ of each other (to remove self-ligated fragments) or were deemed invalid using a computational procedure developed for analyses of $\mathrm{Hi}-\mathrm{C}$ read data [21]. We calculated the efficiency of the restriction enzyme MboI as $71 \%$, using quantitative PCR (Table S3 in Additional file 1). The promoter capture efficiency, i.e., the percentage of aligned reads mapping on targeted promoter regions (which constituted $0.4 \%$ of the genome), was estimated to be 18-44\% (Table S4 in Additional file 1), corresponding to 45-110-fold read enrichment at promoters.

\section{High-resolution mapping of promoter-anchored interactions}

To identify genome-wide promoter-anchored interactions, i.e., interactions with one read mapping to a targeted promoter region and its pair mapping elsewhere in the genome, we required the interactions to be supported with three or more reads in both biological replicates (Fig. S4 in Additional file 2). This resulted in the identification of 94,943 interactions involving 15,905 promoters (corresponding to 12,874 genes) and 71,985 distal regions. (Tables S5 and S6 in Additional file 1). Hereafter, we refer to the genomic regions observed to interact with one or more promoters as distal regions. First, we determined to what extent the resolution to call promoter-anchored interactions was improved with HiCap over previous methods that were based on either sonication (ChIA-PET) or a 6-cutter $(\mathrm{CHi}-\mathrm{C})$. To this end, we compared the lengths of our promoter and distal regions with those identified in published ChIA-PET interaction data generated with RNA polymerase II immunoprecipitation [15, 22] and Capture-Hi-C data in mESCs [17]. The 4-cutter resulted in much higher resolution for both the promoter and distal regions. The promoter fragments used for sequence capture were significantly shorter (mean 885 bp) compared with 6879 bp in a recent study [17] (Fig. 1a), which increased our ability to identify promoter-anchored interactions with proximal enhancers, e.g., those residing within the larger HindIII fragments. For distal regions, ChIA-PET and Capture-Hi-C data had a mean fragment length of 3789 and $3444 \mathrm{bp}$, respectively, whereas HiCap had significantly $\left(P<2.2 \times 10^{-16}, \chi^{2}\right.$ test $)$ shorter fragments (mean 699 bp) (Fig. 1b), an adequate resolution to start mapping individual enhancers. However, it is important to note that ChIA-PET is designed to identify interactions mediated by protein complexes and the difference in resolution is thus compensated for by the specificity of the interaction information obtained. Visualizing the promoter-anchored interactions obtained for three genes (Sco2, Arsa and Shank3) in mESCs with our 4-cutter strategy and a recent 6-cutter study [17] illustrated the benefits of increased fragment resolution (Fig. 1c). HiCap could distinguish between four promoteranchored interactions (coming from three different genes) targeting four closely located regions (Fig. 1d) that were indistinguishable using a 6-cutter strategy. We observed hundreds of similar examples in which multiple HiCap distal regions were found within HindIII fragments used in $\mathrm{CHi}-\mathrm{C}$, as expected (Fig. S5 in Additional file 2). Likewise, using 6-cutter strategies for promoter-anchored 
a

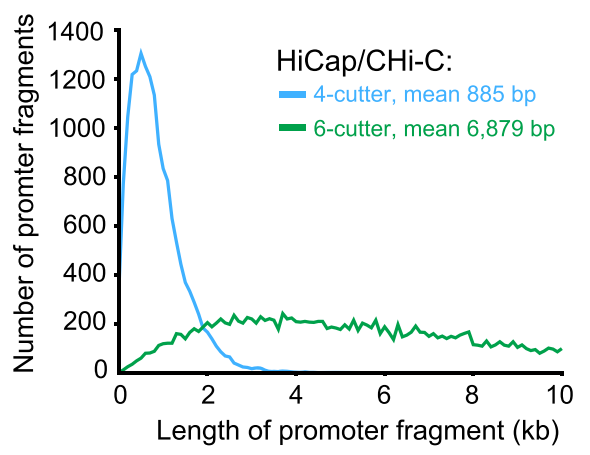

b

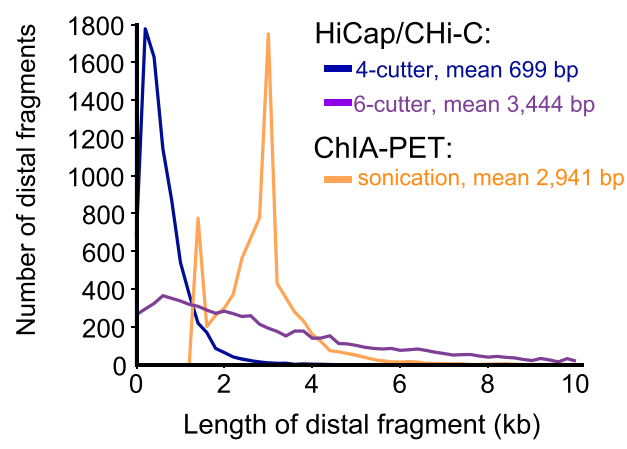

C
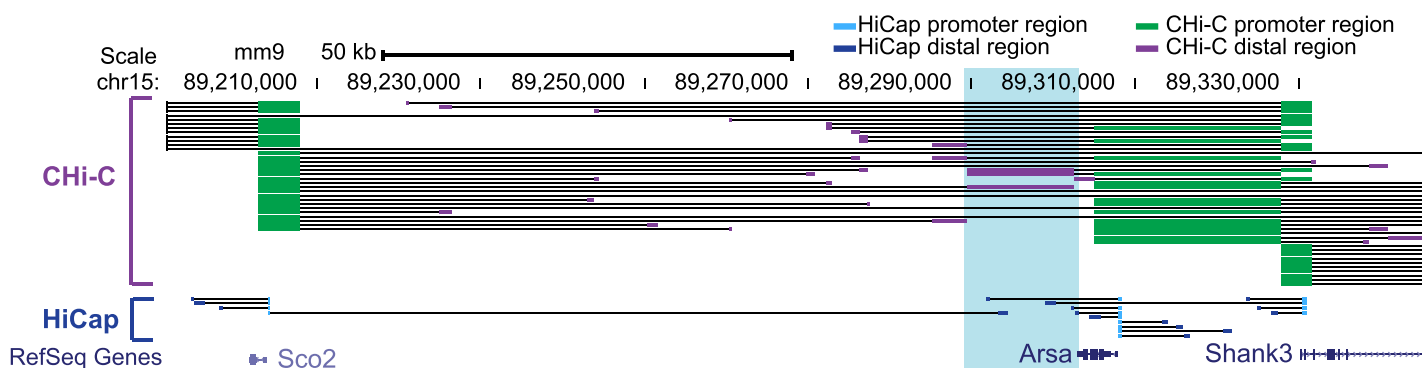

RefSeq Genes

I. Sco2

H3K4me1
H3K27Ac _.

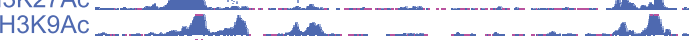

$\mathrm{DHS}-\cdots$

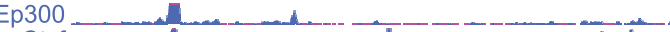

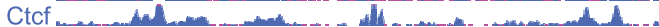

2.

e

\section{Expressed genes}

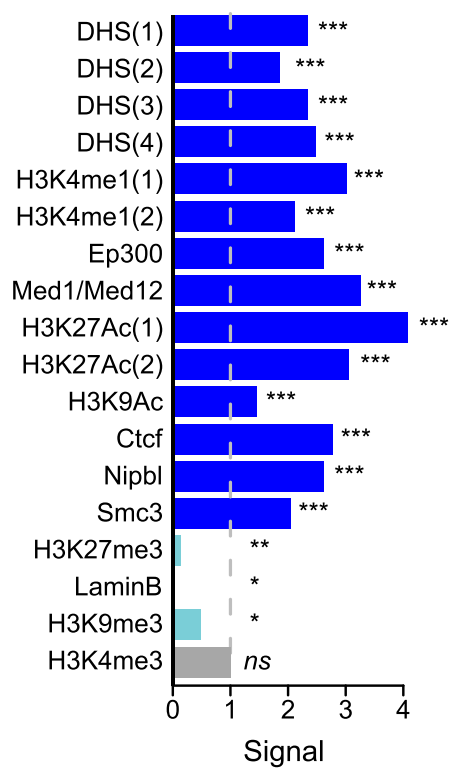

Silent genes

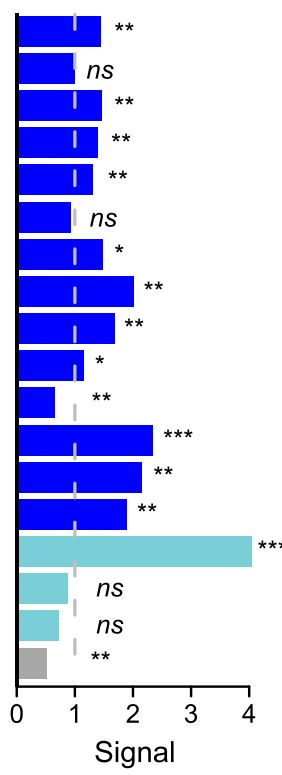

It

d

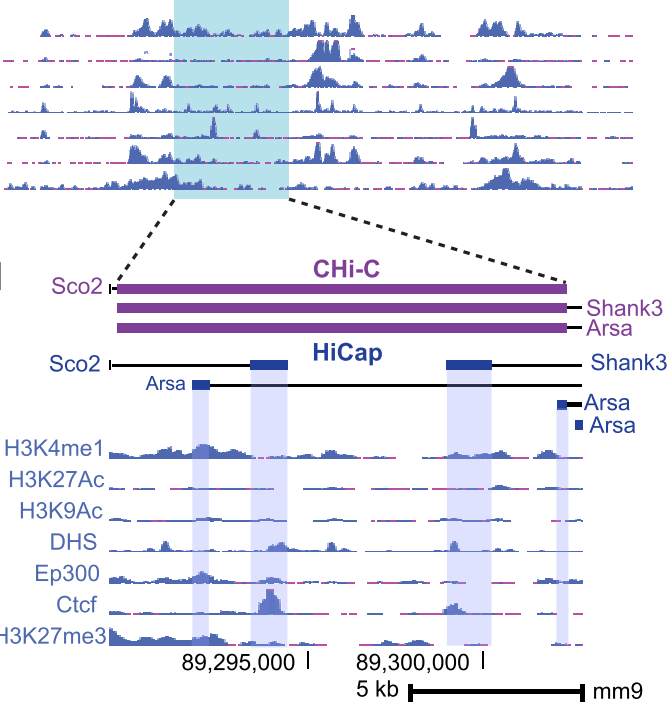

f

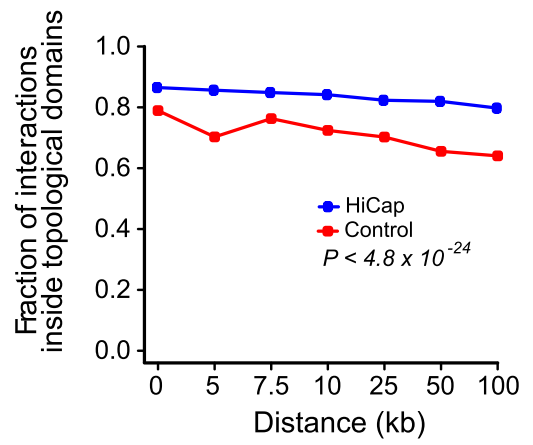

Fig. 1 (See legend on next page.) 
(See figure on previous page.)

Fig. 1 Genome-wide promoter-enhancer mapping with HiCap. a Length distribution of sequence-captured promoter regions in mESCs by HiCap and $\mathrm{CHi}-\mathrm{C}$ based on either a 4-cutter (turquoise) or a 6-cutter (green). b Length distribution of distal regions identified by HiCap/CHi-C 4-cutter (blue), 6-cutter (purple) and ChIA-PET (orange) in MESCs. c Snapshot of observed promoter-anchored interactions for three genes (Sco2, Arsa and Shank3) in HiCap and $\mathrm{CHi}-\mathrm{C}$ data, overlaid with genome-wide enhancer and chromatin marks. $\mathbf{d}$ Detailed zoom-in on one distal region identified by HiCap/CHi-C (6-cutter), which contains multiple smaller distal regions identified by HiCap/CHi-C (4-cutter). Promoters and distal regions are color coded as in $(\mathbf{a}, \mathbf{b})$. Gene names indicate which gene the distal region is interacting with. In both cases, these distal regions are interacting with same genes (ScO2, Arsa and Shank3). e Signal (observed overlap divided by expected) between HiCap promoter-anchored interactions mapping to distal regions and published genome-wide enhancers (blue), chromatin marks for silent genes (turquoise) and promoter marks (gray) in mESCs. HiCap distal regions were classified into expressed [ $>3$ RPKM (reads per kilobase of gene model and million uniquely mapped reads)] and silent ( $\leq 0.3$ RPKM) by the expression of their target genes. Significant $\left(X^{2}\right.$ test) comparisons are indicated with asterisks: ${ }^{*} P<0.05$, ${ }^{* *} P<0.001$; ${ }^{* * *} P$ $<10^{-10}$; ns not significant. $\mathbf{f}$ Fraction of observed HiCap interactions contained within topologically associating domains (TADs), as a function of the interaction distance and compared with expected

interactions might be complementary as they, by design, identify longer-range interactions.

\section{HiCap interactions are enriched for regions with enhancer features}

In order to characterize the high-resolution promoteranchored HiCap interactions, we investigated to what extent the HiCap distal regions overlapped with those enriched with enhancer-associated features from ChIP-seq and DNase hypersensitivity experiments (Table S7 in Additional file 1), henceforth referred to as "putative enhancers". We excluded promoter-promoter interactions for these analyses. Overall, $64 \%$ of the promoteranchored HiCap distal regions overlapped putative enhancers, and we next assessed the enrichment of specific enhancer features in the distal regions as the ratio of observed to expected overlap. Expected overlaps were computed through randomly sampling fragments from annotated promoters using the observed distance distributions of HiCap interactions (preserving the non-random locations of promoters and enhancers in our background model). We found that HiCap distal regions interacting with promoters of expressed genes [RPKM (reads per kilobase of gene model and million uniquely mapped reads) $>3$ ] were significantly $\left(P<10^{-21}\right.$, Chi-square $\left(\chi^{2}\right)$ test) enriched for putative enhancers (Fig. 1e) carrying active marks, and significantly depleted for chromatin regions carrying repressive marks such as H3K27me3, Lamin B1 and H3K9me3 $\left(P=9.6 \times 10^{-8}\right.$, $P=9.2 \times 10^{-8}$ and $P=0.014$, respectively, $\chi^{2}$ test $)$. Moreover, these distal regions were not enriched $(P=0.86$, $\chi^{2}$ test) for promoter-associated H3K4me3 marks. In contrast, distal regions connected to promoters of transcriptionally silent genes were strongly enriched for the repressive chromatin mark H3K27me3 $\left(P=3.3 \times 10^{-13}\right.$, $\chi^{2}$ test; Fig. 1e). Furthermore, regions interacting with negative controls were significantly depleted for enhancerassociated chromatin marks and showed a significant enrichment for repressive chromatin marks (Fig. S6 in Additional file 2). Overall, these results demonstrate that the promoter-anchored interacting regions were highly enriched for regions with enhancer-associated chromatin marks or protein complex binding. As expected, we also observed that most HiCap interactions were contained within the same topologically associating domains (TADs; $P<4.8 \times 10^{-24}, X^{2}$ test) and they were also depleted outside TADs $\left(P<3.2 \times 10^{-171}, \chi^{2}\right.$ test $)$ (Fig. 1f; Fig. S7 in Additional file 2).

\section{Expression of enhancer RNA from mapped distal regions}

We observed that HiCap distal regions were often expressed; e.g., $30 \%$ had expression above 1 RPKM (Fig. 2a). Moreover, distal regions were significantly more often expressed than random intergenic regions within the same distances from promoters $\left(P<2.2 \times 10^{-16}\right.$, Wilcoxon rank sum test; Fig. 2a). Importantly, HiCap distal regions connected to active promoters had significantly higher expression levels than those connected to silent promoters $\left(P=6 \times 10^{-45}\right.$, Wilcoxon rank sum test; Fig. 2b). We also observed that the expression levels of distal regions and the connected gene with a HiCap interaction mapped to its promoter were more highly correlated ( $P=0.001$, permutation test; Fig. $2 \mathrm{c}$ ) than the closest genes of distal regions without a HiCap connection (Fig. 2d). Furthermore, the expression of HiCap distal regions connected to non-closest genes also showed significantly higher correlation $(P=0.001$, permutation test; Fig. 2e) than random non-closest genes on the same chromosome at the same distance apart (Fig. 2f). In conclusion, both enhancer-associated chromatin marks and enhancer RNA expression were found at HiCap distal regions in support of HiCap enrichment for promoteranchored interactions involving enhancer regions.

\section{HiCap interactions predict differentially expressed genes upon TF over-expression}

Although several studies have mapped genome-wide chromatin interactions [11-15], it remains to be determined whether the interactions are sufficiently enriched for bona fide regulatory interactions to be predictive of gene expression levels, in particular in comparison with 
a

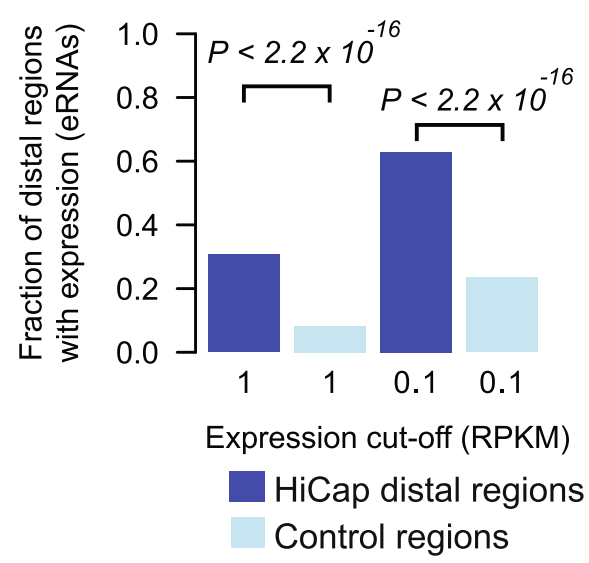

c

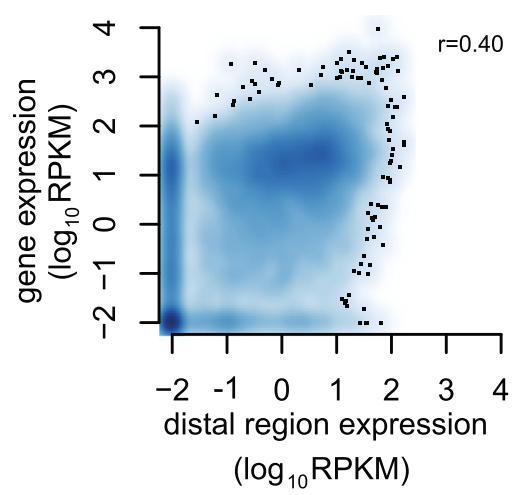

e

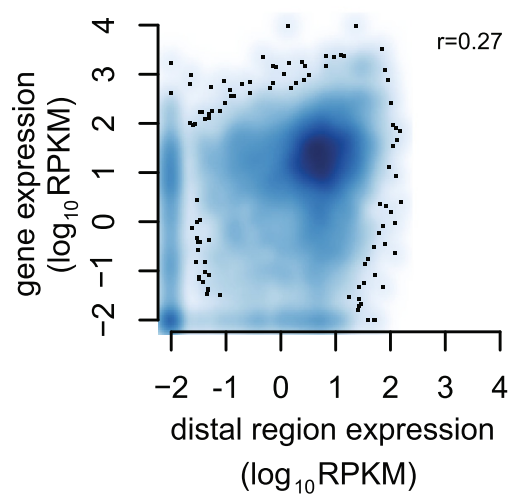

b

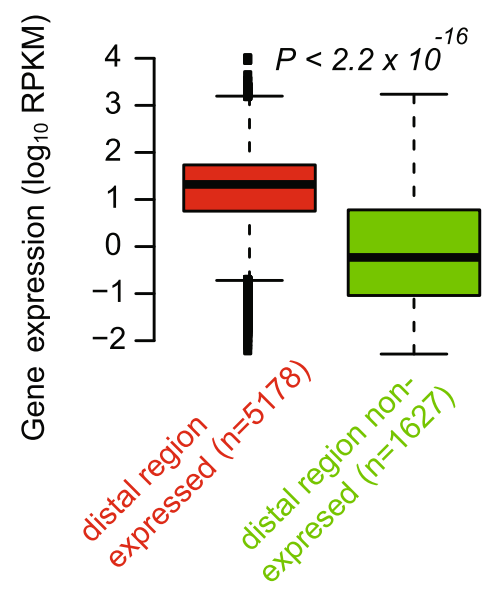

d

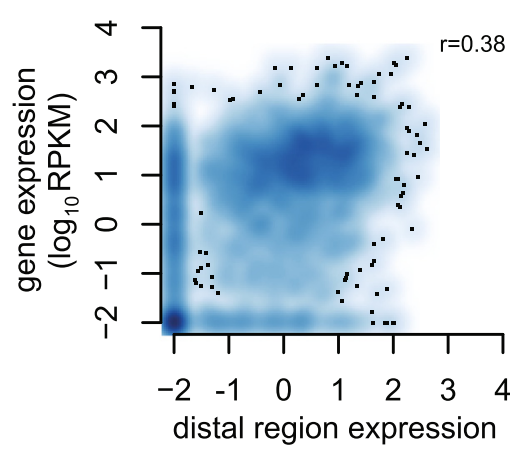

$\left(\log _{10}\right.$ RPKM)

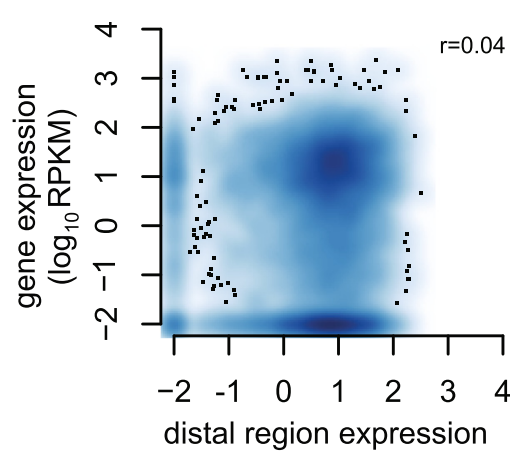

$\left(\log _{10}\right.$ RPKM)

Fig. 2 Expression of HiCap-identified distal regions and their correlations with target gene expression. a Fraction of HiCap distal regions with expression above 0.1 or 1 RPKM compared with random regions sampled within the same distances from promoters as observed interactions. b Boxplot comparing the expression of genes connected to either highly expressed (>10 RPKM) or non-expressed ( $\leq 0.3$ RPKM) distal regions; number of genes is given in parentheses. c Spearman correlation of the expression of HiCap-mapped distal regions and their closest HiCap connected target genes. $\mathbf{d}$ Spearman correlation of the expression of HiCap-mapped distal regions and the closest gene without HiCap interaction. e Spearman correlation of expression of HiCap distal regions and non-closest HiCap connected target genes. $\mathbf{f}$ Spearman correlation of expression levels of HiCap distal regions and the expression of randomly connected non-closest genes on the same chromosome. When multiple distal regions are connected to the same gene, the RPKM sum was used for analyses in (b-f) 
the current best practice, which is to link enhancers to their closest genes. To this end, we re-analyzed genomewide binding locations of 15 different TFs in mESCs together with genome-wide differential expression analyses after TF overexpression to determine whether genes with HiCap interactions to putative enhancers were more often found upregulated. We first focused on the closest genes to mapped TF binding sites (Fig. 3a) and found that genes with HiCap interaction support for mapped TF binding sites were more often upregulated than those without HiCap interaction support (Fig. 3b). The higher enrichment was significant $(P<0.001$, Fisher's exact test $)$ for HiCap interactions at several read thresholds (Fig. 3b). There was also a trend for published $\mathrm{Hi}-\mathrm{C}$ interactions [12] to agree with the overexpression data.

To investigate the functional relevance of interactions between distal regions and non-closest genes they are connected to, we evaluated their enrichment for upregulated genes. HiCap interactions mapped to more distant (non-closest) genes had similar and sometimes even higher enrichment for upregulated genes than the set of closest genes (Fig. 3c, d). Linking distant genes using the $\mathrm{Hi}-\mathrm{C}$ dataset, however, resulted in worse enrichment than the closest gene set. Passing this rather strict perturbation-based validation test (strict since the effect sizes were compared with the effects for closest genes which often are targets) gives confidence that HiCap interactions reflect TF and, by extension, enhancer action.

\section{Network analyses of HiCap interactions}

Most distal regions interacted with only one promoter (1.32 promoters on average), whereas the promoters interacted with 5.97 distal regions on average, often within 1-100 kb and both degree distributions followed a power-law indicative of a robust network topology [23] (Fig. 4a, b). Since gene regulation in the nucleus has a spatial component $[24,25]$, we investigated whether global HiCap interactions could inform about general organization of regulatory interactions. We noted an apparent enrichment for interconnected clusters (cliques) of only promoters (Fig. 4c, d), only distal regions (Fig. 4e, f) as well as motifs involving both distal regions and promoters (Fig. $4 \mathrm{~g}-\mathrm{k}$ ). The largest promoter cliques we found involved 19 promoters each, all involving a group of genes on chromosome 17 (Fig. 4l). Interactions involving two promoters were likely overrepresented due to sequence capture. But we also detected high read support for interactions involving two distal regions, which surprised us considering these regions were not enriched for by sequence capture. We did rediscover those interactions in our 4-cutter $\mathrm{Hi}-\mathrm{C}$ data (Fig. 4m; Fig. S8 in Additional file 2). Moreover,

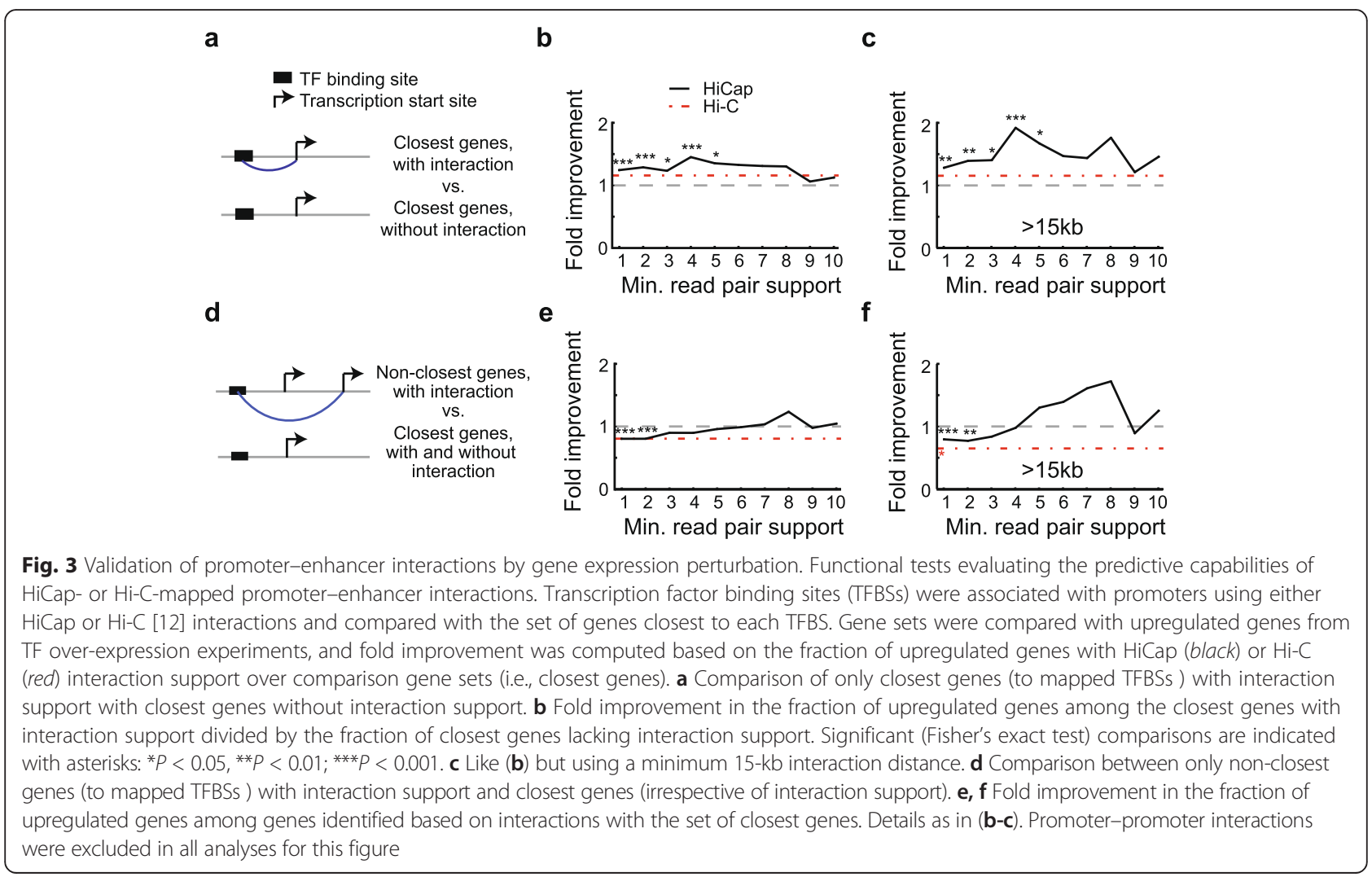


a

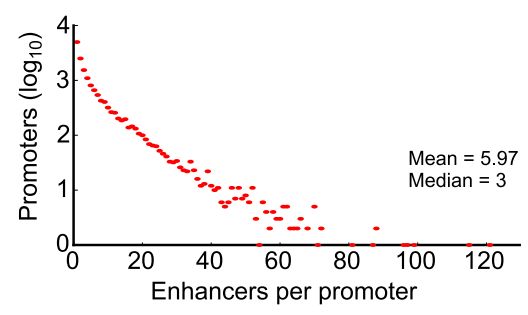

b

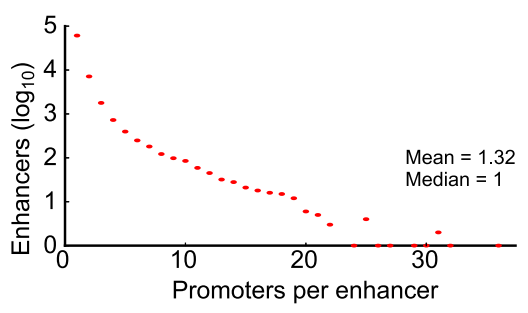

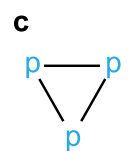

d

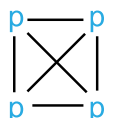

347,550

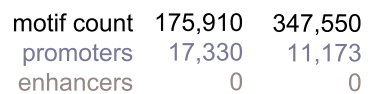

enhancers 00

enrichment $397 x \quad$ inf

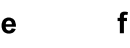<smiles>o1oo1</smiles><smiles></smiles>

g<smiles>o1o[pH]1</smiles>

h

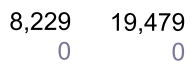

$\begin{array}{rr}0 & 0 \\ 3,343 & 1,132\end{array}$

$40 x \quad 48,697 x$

with promoters

motif count $\quad 119,166 \quad 242,661$ enrichment $270 \mathrm{x} \quad \sim$ inf

$8,229 \quad 19,479$

$40 x \quad 48,697 x$

m
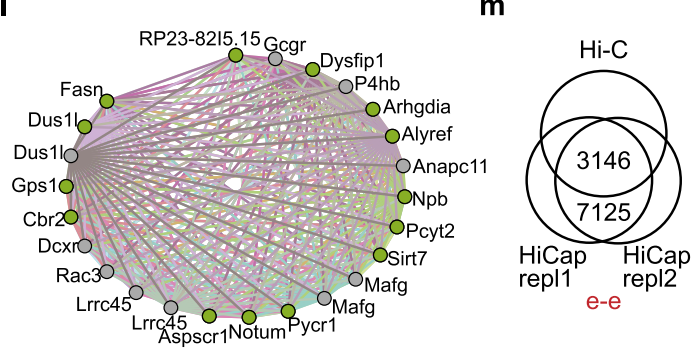

n

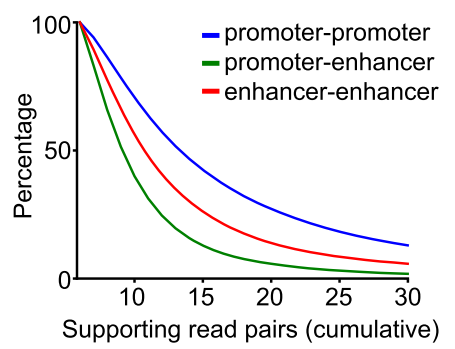

$q$

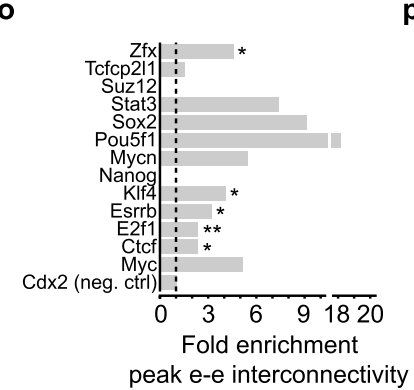

p

Number of significant GO terms (5\% FDR)
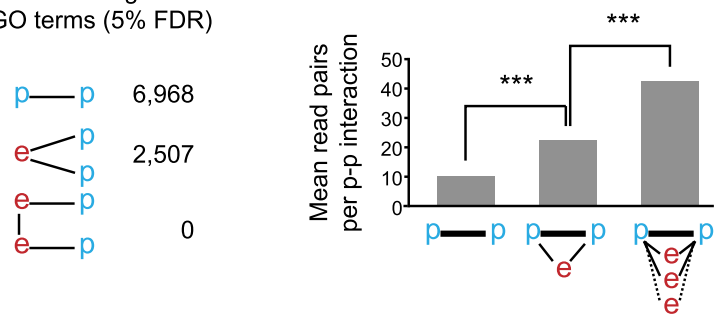

Fig. 4 Motifs and interconnected clusters of promoters and enhancers. $\mathbf{a}$, b Distributions of interaction for promoters (a) and enhancers (b). c-k Interaction motifs involving promoters $(p)$ and/or enhancers (e) together with their occurrence among HiCap interactions and the numbers of unique promoters and enhancers within the motifs. I The most highly interconnected set of promoters, arranged by genomic coordinates and annotated with gene name. Promoters in green are all connected to each other whereas the ones in grey are missing some interactions. $\mathbf{m}$ Overlap between enhancer-enhancer interactions identified in HiCap and in-house Hi-C interactions. $\mathbf{n}$ Percentage of interactions supported with different read pair thresholds. o Mean degree for nodes (i.e., enhancers) in subnetworks of only enhancer nodes bound by the same TF or chromatin remodeling protein. Asterisks indicate significance $\left.{ }^{*} P<0.05,{ }^{*} P<0.01,{ }^{* *} P<0.001\right)$ computed against randomized enhancer node selections in the network. $\mathbf{p}$ Number of significant gene ontology $(G O)$ categories enriched among gene pairs that share the same category, for genes connected by HiCap interaction motifs. FDR false discovery rate. $\mathbf{q}$ Mean read pair support for promoter pairs with increasing number of common enhancer interactions $(0,1$ or $\geq 2)$

read support for interactions involving two distal regions was higher than for interactions between a promoter and a distal region (Fig. 4n), providing additional support for enhancer-enhancer interactions [12] and indicating that they are prevalent (Table S6 in Additional file 1). Further analyses of the interactions between distal regions revealed that they were more often bound by the same TF than what would be expected by chance, with significant 
enrichments for Zfx, Klf4, Essrb, E2f1 and Ctcf, and a trend towards enrichment for other factors (Fig. 4o). Interestingly, genes connected through promoter interactions or mutual interactions to the same distal regions were more often annotated to belong to the same gene ontology categories (Fig. 4p; Table S8 in Additional file 1), supporting the previous finding that such interactions could be involved in transcriptional coordination [15]. Finally, we observed that pairs of genes with interacting promoters had, on average, higher read pair support if they were additionally interacting with one or more distal regions (Fig. 4q).

\section{Discussion}

We developed HiCap for the genome-wide identification of regulatory interactions. It was important to base the method on $\mathrm{Hi}-\mathrm{C}$, rather than $3 \mathrm{C}$ as in Capture-C, to obtain sufficient enrichment for ligated fragments. Using HiCap, we observed stronger enrichments for ChIP-seq inferred enhancers than with existing methods, likely due to incorporation of sequence capture targeting promoter sequences. Also, HiCap provides better sensitivity compared with $\mathrm{Hi}-\mathrm{C}$ by fixing one interaction partner, thereby overcoming the need to quadruple the sequencing depth to double the sensitivity. Considering $4 \mathrm{C}$ as a reference method with the highest resolution to identify interactions at selected loci, HiCap had comparable reproducibility to other genome-wide methods (Fig. S9 in Additional file 2). It would be possible to use a broader target set such as the $\sim 600,000$ Fantom 5 putative promoters [26] (8.7 \% of which are within our promoter set), although it might not be possible to fit such numbers onto current oligo synthesis arrays. A recent study [27] introduced a combination of $\mathrm{Hi}-\mathrm{C}$ with selected sequence capture of three long genomic regions containing diseaseassociated SNPs in gene deserts to identify several interactions in breast cancer. This illustrates the flexibility in combining $\mathrm{Hi}-\mathrm{C}$ with sequence capture probes for disease-associated regions in detail [27] or genomewide promoter interactions as performed in HiCap (this study) and Capture-Hi-C [17, 18]. Although increased resolution allowed HiCap to identify distal regions within $\mathrm{CHi}-\mathrm{C}$ bait and distal regions, it is important to note that using a 6-cutter during the Hi-C step enabled $\mathrm{CHi}-\mathrm{C}$ to identify longer range interactions compared with HiCap, which employs a 4-cutter during the Hi-C step (Fig. S10 in Additional file 2). Since HiCap relies on promoter capture, it may not be well-suited for studying organisms where the promoter regions are not well-annotated.

The promoter-anchored chromatin landscape did not just interact with distal regions. We observed extensive promoter-promoter interactions, but also more surprisingly abundant interactions between two or more distal regions. Despite our enrichment for promoter-anchored interactions, which selected against such distal to distal region interactions, we observed them with comparable read support to promoter-anchored distal interactions. This result supports an early $\mathrm{Hi}$-C-based observation of putative enhancer-enhancer interactions in mESCs [13], and indicates that interconnected enhancer regions might be interesting to explore functionally. The distal regions seem to be enhancers, as they interact with promoters and are usually occupied by enhancer-associated TFs. Additionally, our analyses indicate that interacting pairs of distal elements are enriched for occupation by the same TF, which might help explain the formation or function of these interactions.

Although a large fraction of distal regions (65\%) were connected to the closest gene, HiCap identified thousands of long-range interactions. Importantly, we demonstrated that interactions between distal regions and more distant (non-closest) genes were as enriched for genes that became upregulated after TF over-expression as the set of closest genes. Although our improvement has a modest effect size, our results suggest that target genes from ChIP-seq experiments should contain both closest genes together with HiCap interactions involving genes further away from the TF binding location without diluting the signal. Similar incorporation of Hi-C interactions would dilute signal and should be avoided. TF perturbation tests, such as the one introduced in this study, will be important to assess predictive abilities of interactions identified in existing and novel methods. At present, it demonstrates that regulatory interactions are significantly captured with HiCap, but at the same time that predictive power is modest.

\section{Conclusions}

We describe a new strategy for high-resolution mapping of genome-wide chromatin interactions anchored on promoters. In order for our resolution to match the sizes of promoters and enhancers, we shifted from using a 6-cutter restriction enzyme to instead using a 4-cutter. This resulted not only in higher resolution of promoters and distal regions, but also higher enrichment for enhancer features in our distal regions than has been reported in previous studies. Therefore, the methodology developed in this study will be important for high-resolution characterization of genome-wide interactions involving promoters and enhancers.

\section{Materials and methods Culturing of $\mathrm{mESCs}$}

mESCs (line R1) were obtained from Janet Rossant's lab (Toronto, Canada). Cells were maintained on $0.1 \%$ gelatin-coated dishes in Dulbecco's modified Eagle medium (DMEM) supplemented with $10 \%$ fetal calf 
serum, $0.1 \mathrm{mM}$ non-essential amino acids, $0.3 \mathrm{mg} / \mathrm{ml}$ L-glutamine, $1 \mathrm{mM}$ pyruvate (Invitrogen), and $1000 \mathrm{U} /$ $\mathrm{ml}$ murine leukemia inhibitory factor (Chemicon International ESGRO), and were kept in a $5 \% \mathrm{CO}_{2}$ atmosphere at $37{ }^{\circ} \mathrm{C}$. The medium of undifferentiated cells was changed daily.

\section{Experimental procedure of HiCap}

$\mathrm{Hi}-\mathrm{C}$ was performed on mESCs as previously described [11], except for the following modifications. We generated replicate experiments from $\sim 5$ million mESCs that were cross-linked with $1 \%$ formaldehyde for $10 \mathrm{~min}$. Cells were lysed and nuclei were isolated. Isolated nuclei were digested with 4-cutter FastDigest MboI (Thermo Scientific, $1 \mu \mathrm{l} / \mu \mathrm{g}$ DNA) for $4 \mathrm{~h}$ at $37^{\circ} \mathrm{C}$. The ends of digested material were filled with biotinylated dATP, dGTP, dCTP and dTTP using Klenow fragments (Fermentas, $0.1 \mathrm{U}$ per $1 \mu \mathrm{g}$ DNA). Klenow was deactivated using $0.01 \mathrm{M}$ EDTA at $75{ }^{\circ} \mathrm{C}$ for $15 \mathrm{~min}$. Then the material was diluted to $3.5 \mathrm{ng} / \mu \mathrm{l}$ and ligated using $\mathrm{T} 4$ DNA Ligase (Promega). The crosslinking was reversed by adding Proteinase $\mathrm{K}$ and incubating overnight at $65^{\circ} \mathrm{C}$. The proteins were removed and DNA was purified using phenol-chloroform followed by ethanol precipitation. Biotinylated but unligated ends were removed using T4 DNA polymerase by incubating at $12{ }^{\circ} \mathrm{C}$ for $15 \mathrm{~min}$. The material was fragmented to $300-600$ bp by sonication. The fragment ends were repaired and A-tailed. Then the biotinylated fragments were bound to streptavidin beads and unbound fragments were washed away. Sequencing adapters were then ligated to the fragments bound to beads. The material was amplified for six to nine cycles while bound to beads to obtain sufficient amounts for sequence capture. Original biotinylated material was removed and the supernatant was hybridized to a sequence capture probe set according to the manufacturer's instructions (Roche Nimblegen Inc.). Hybridized material was washed according to the manufacturer's instructions and amplified with PCR for three to six cycles.

Hybridization of the probes to the $\mathrm{Hi}-\mathrm{C}$ material was done exactly according to the manufacturer's instructions (Roche Nimblegen Inc). Briefly, $1 \mu \mathrm{g}$ of $\mathrm{Hi}-\mathrm{C}$ material was mixed with $5 \mathrm{mg}$ COT DNA, $1 \mu \mathrm{l}$ of $1000 \mu \mathrm{M}$ Universal Oligo, and $1 \mu \mathrm{l}$ of $1000 \mu \mathrm{M}$ Index Oligo and dried down in a vacuum concentrator on high heat $\left(60^{\circ} \mathrm{C}\right)$. Then, 7.5 $\mu \mathrm{l}$ of $2 \times$ hybridization buffer and $3 \mu \mathrm{l}$ of hybridization component A [these components are included in the Nimblegen SeqCap EZ Hybridization and Wash Kit (cata$\log$ number 05634261001 )] were added to the dried down material, mixed well by vortexing for $10 \mathrm{~s}$ and centrifuged for $10 \mathrm{~s}$. The mix was placed in a $95{ }^{\circ} \mathrm{C}$ heat block for 10 min to denature the DNA, and then centrifuged for $10 \mathrm{~s}$ at maximum speed. The mixture was then transferred to a $0.2 \mathrm{ml}$ PCR tube containing $100 \mathrm{ng}$ of the appropriate probe set ( $4.5 \mu \mathrm{l}$ volume). The mixture was vortexed for $3 \mathrm{~s}$ and centrifuged for $10 \mathrm{~s}$ and placed in a thermocycler set at $47^{\circ} \mathrm{C}$ for incubation for $64-72 \mathrm{~h}$. The thermocycler's heated lid was set to $57^{\circ} \mathrm{C}$.

After the incubation, the mixture was washed to eliminate unhybridized probes. Wash buffers (Stringent, I, II and III) and $100 \mu \mathrm{l}$ of streptavidin beads were prepared for each hybridization according to the manufacturer's instructions. The hybridization mix was mixed with 100 $\mu \mathrm{l}$ of streptavidin beads, further mixed by pipetting up and down 10 times and placed back in the thermocycler at $47{ }^{\circ} \mathrm{C}$ for $45 \mathrm{~min}$. After the incubation, $100 \mu \mathrm{l}$ of $1 \times$ wash buffer I heated to $47^{\circ} \mathrm{C}$ was added to the mix and vortexed for $10 \mathrm{~s}$. The contents of the tube were transferred to a $1.5 \mathrm{ml}$ tube that was placed in a magnet to bind the beads. The liquid was removed and discarded once clear. Stringent wash buffer $(200 \mu \mathrm{l}, 1 \times)$ heated to $47^{\circ} \mathrm{C}$ was added to the beads, pipetted up and down 10 times and incubated for $5 \mathrm{~min}$ at $47{ }^{\circ} \mathrm{C}$. The mix was then placed in the magnet and liquid was removed once clear. The wash with $1 \times$ Stringent wash buffer was repeated once more. Then, $200 \mu \mathrm{l}$ of $1 \times$ wash buffer I was added to the mixture and mixed by vortexing for 2 min; the beads were collected using the magnet and liquid was discarded once it was clear. The same steps were then repeated using $300 \mu \mathrm{l}$ wash buffer II (except this time vortexing for $1 \mathrm{~min}$ ) and $200 \mu \mathrm{l}$ wash buffer III (except this time vortexing for $30 \mathrm{~s}$ ). To elute the captured material from the beads, $50 \mu \mathrm{l}$ of PCR-grade water was added to the beads and they were stored at $-20{ }^{\circ} \mathrm{C}$ until further use.

The resulting DNA libraries were sequenced $100 \mathrm{bp}$ from both ends (paired-end sequencing) on a HiSeq 2000 (Illumina Inc.). This is long enough to map to 90 \% of the genome [28], including, e.g., dead retrotransposon repeats $[87 \%$ mappability for long terminal repeats, $82 \%$ for long interspersed elements (LINEs), $98 \%$ for short interspersed elements (SINEs)], as annotated by RepeatMasker and using mappability files from MULTo [28]. We performed a number of alternative washing procedures to see if we could improve sequence capture efficiency. However, we find that the washing procedure recommended by the manufacturer performed the best. Table S9 in Additional file 1 summarizes the alternative washing procedures tried and corresponding sequence capture efficiency (percentage of reads that are mapped on the probe sequences).

\section{Mapping of sequence data}

Paired-end sequences were aligned to the mouse genome (build mm9) through HiCUP [21] which used Bowtie [29] version 0.12.7 in single-end mode for the two ends separately, and with iterative trimming from 
the 3' end for unaligned reads. Multi-mapping reads were discarded. Paired-end mapping is not suitable for HiCap libraries as the 100 bp on either end often contain the ligation point so that a paired-end mapper would soft trim that sequence end, effectively removing the pairing information. We therefore used custom scripts to pair the independently mapped sequence ends and we indexed each sequence pair to their corresponding MboI restriction fragment.

\section{Sequence capture probes}

We designed sequence capture probes against mouse promoters compiled from multiple sources. RefSeq and Ensembl annotations were used together with transcription start sites from DBTSS (from 25 May 2010) and MPromDb (from 28 May 2010). There were in total 53,501 target sequences (targeting closest upstream and downstream MboI sites of each promoter and negative controls) and the probes covered $93.5 \%$ of the target bases (11,293,801 bases). DBTSS is based on full-length mRNAs, and mostly corresponds to RefSeq and Ensembl. MPromDb is based on RNA polymerase II and H3K4me3 ChIP-seq data for different cell types, including ESCs. From annotated transcript start sites, we searched for the closest restriction cut sites (GATC) on each side, and chose the last 150 bp before the cut site as the captured regions. When restriction sites were $<300 \mathrm{bp}$ apart we chose the whole region between them. From these regions, Nimblegen designed the actual probe sequences. We also selected exonic and intergenic control regions which were included in the same probe selection pipeline.

\section{Calling of interactions}

HiCUP software available at Babraham Bioinformatics [21] was used to filter out non-informative and unlikely pair combinations. Read pairs with the exact same mapping positions were discarded (to remove any potential effect from PCR duplicates) and pairs less than 1 $\mathrm{kb}$ apart were excluded. We only used pairs with at least one read mapping to probe regions. We counted the number of times each pair is observed for each set of probes belonging to promoters to derive interaction read support. We required at least three supporting read pairs in each biological replicate to call an interaction. We did not see any correlation between the number of restriction enzyme fragments closest to a transcription start site and interactions originating from that transcription start site $\left(\mathrm{r}^{2}=0.065\right.$, Pearson correlation coefficient). $\mathrm{G}+\mathrm{C}$ content of HiCap distal regions was slightly higher than the genome average ( $47 \pm 6.7)$; $40-70 \%$ for $99 \%$ of HiCap distal regions. Promoterpromoter interactions were called similarly, but required that both ends of the paired reads aligned with probes belonging to promoters. We also mined the raw read pairs for interactions involving only distal regions. For this purpose we collected all distal regions from significant promoter-distal interactions and performed similar analyses for read pairs with both ends originating from a HiCap distal region. Following is a breakdown of called interactions and how they distribute over expressed and non-expressed genes. We detected at least one interaction for $73 \%$ of the expressed genes $(11,786$ out of 16,241 , RPKM $>0.3)$ and for $48 \%$ of genes with no detectable expression (6532 out of 13,584). Unsaturated sequencing could account for the fact that we did not detect any interaction for $27 \%$ of the expressed genes. It is not surprising, however, that we did not detect any interaction for $52 \%$ of genes with no expression as they might not be involved in distal interactions. There are also cases where only one of the alternative promoters of the same gene is involved in a distal interaction; therefore, it is fairer to assess the number of genes with interactions rather than the number of promoters.

\section{Analyses of overlap with enhancer ChIP-seq data}

We downloaded enhancer regions inferred from different ChIP-seq experiments carried out in mESCs (Table S7 in Additional file 1). We sorted the mapped regions in each experiment to analyze only the top 5000 mapped regions from each experiment, in order to control for different signals and background levels in the different experiments. For Mediator data, we downloaded raw reads for Med1 (SRX022694 and SRX022695) and Med12 (SRX022692 and SRX022693) and aligned these to the mouse genome $\mathrm{mm} 9$. We performed peak calling using SISSRs version 1.4, and concatenated and sorted the peaks. ChIP-seq mapped regions were extended to $1000 \mathrm{bp}$ if they were shorter (relevant only for Mediator bound regions). For analyses of HiCap overlap with putative enhancers, we computed the observed to the expected overlap. To calculate the percentage of HiCap promoter-enhancer interactions overlapping with at least one enhancer mark we simply overlapped HiCap enhancers with Chip-seq associated mESC enhancer features from Additional file 1: Table S7. The observed overlap was simply computed as the fraction of HiCap interactions that overlapped (by at least one nucleotide) with enhancer mapped regions. To compute the expected overlap we randomly sampled regions close to annotated transcription start sites, using the actual distance distribution of HiCap interactions. We found this procedure to better control for the non-random locations of genes and enhancers in the genome, whereas the computation of expected overlap based on a fully random model (the fraction of genomic fragments overlapping putative enhancers) rendered all tests significant. 


\section{Comparison of HiCap and in-house Capture-C with pub- lished Capture-C}

We performed Capture-C (3C coupled with sequence capture) using our custom promoter probes. We downloaded Capture-C raw reads from the Gene Expression Omnibys (GEO) database with sample IDs [GEO:GSM1156607] and [GEO:GSM1156608] (for Ter119 ${ }^{+}$cells) and [GEO:GSM1156609] (for mESCs).

\section{Expression level analyses in mESCs}

We prepared a RNA-seq library for mESCs using the Illumina mRNA-seq protocol. The library was sequenced with an Illumina GAIIx at 50 bp read length in single-end mode (Fasteris, Switzerland). Reads were aligned to the mouse genome ( $\mathrm{mm} 9$ assembly) and a comprehensive collection of splice junctions [30] using Bowtie (version 0.12.7). Expression levels were estimated as RPKM using Rpkmforgenes [31], where only uniquely mappable positions were included in the gene model length. Mappability was determined using MULTo [28] and gene models were based on RefSeq annotation downloaded from the UCSC genome browser on 31 July 2011.

\section{Functional test of HiCap interactions}

To assess the power of HiCap interactions to predict differentially expressed genes after TF perturbation, we constructed the following test (with results presented in Fig. 3). TF binding data were downloaded [32] (Table S3 in Additional file 1) as well as expression data after TF overexpression [33] (Table S2 in Additional file 1). For each TF present in both datasets, we listed the closest gene to each midpoint of the binding region. We identified HiCap interactions connecting promoters to the restriction fragment containing the binding site midpoint and listed the genes of those promoters. For Fig. 3b, c, we compared the closest gene of peaks without HiCap interactions with the closest genes that also had a HiCap interaction. For Fig. 3e, f, we compared non-closest genes with HiCap support with the set of closest genes (irrespective of HiCap interactions). This procedure was performed also on $\mathrm{Hi}-\mathrm{C}$ interactions. We compared the fraction of upregulated genes present within the gene sets and report the differences as fold improvements, by dividing the two numbers by one another. To explain the test in detail for Fig. $3 \mathrm{~d}-\mathrm{f}$, we computed the number of closest genes, Nc, and the number of HiCap-connected genes, Nh. From the expression data we identified differentially expressed genes after each TF perturbation independently (false discovery rate $\leq 0.05$ and fold change $>1.5$ ). Next, we computed the number of unique genes that were differentially expressed and also present in either the set of closest genes (Uc) or HiCap inferred (non-closest) genes (Uh). We summarized the enrichment as fold improvement
[Uh/Nh]/[Uc/Nc] and calculated a $P$ value using the $\chi 2$ test with $\mathrm{Uh}$ and $\mathrm{Uc}$ as observed and $\mathrm{Nh} / \mathrm{Nc}$ as their expected ratio. For the compound test including all TFs, we summed all values of Nc, Nh, Uc, and Uh and performed the same tests.

\section{Visualization of interactions}

We downloaded a significant "promoter-other" interaction table for $\mathrm{CHi}-\mathrm{C}$ and selected 548,551 interactions based on their log observed/expected value [17]. We made a GFF file for HiCap and $\mathrm{CHi}-\mathrm{C}$ interactions and uploaded it to the USCS Genome Browser. We overlaid interactions on selected tracks of enhancer features (while keeping their default minimum and maximum data range unchanged).

\section{Analysis of TADs}

We downloaded TAD coordinates from a Hi-C study on mouse ESCs [12]. We then calculated the fraction of HiCap interactions completely contained within a TAD, spanning two or more TADs, or with one or both ends outside annotated TADs. We performed the same analysis on control region interactions that were calculated by randomizing the chromosomes while keeping the distance the same as in HiCap. The fraction of interactions was calculated as a function of the distance between promoters and distal elements. $P$ values were calculated using the $X^{2}$ test on each paired fraction and the highest $P$ value was reported.

\section{Analyses of enhancer RNA expression}

We re-analyzed mapped GRO-seq data present in the GEO (GSM1186440 and GSM1186441 combined) [34] to determine expression levels for HiCap-inferred distal regions. For that we used HiCap distal regions that do not overlap (intergenic, $42 \%$ ) with any genes from the RefSeq annotation. In parallel, we generated random regions located within the same distances from promoters as HiCap distal regions, having the same average length as HiCap distal regions and not overlapping with genes from RefSeq annotation. We calculated expression levels (RPKM) for distal and random regions using the Rpkmforgenes [31]. P-values were computed using $X^{2}$ test based on the fraction of total regions from HiCap and random with expression above either 0.1 or 1.0 RPKM.

\section{Interaction motifs}

We mined the HiCap interactions between promoters and enhancers to enumerate the occurrences of motifs (Fig. 4a-j). As a background model to calculate enrichment, we randomized promoter-promoter, enhancerpromoter and enhancer-enhancer interactions separately five times but keeping the degree distributions. 
Motifs which did not occur in the background were assigned " $\sim$ inf" enrichment. To calculate $P$ values in Fig. 4q, we grouped interactions by distance (1000-1999, 2000-3999, 4000-7999, etc. up to $64,000-127,999)$ and by the sum of the degree of the promoter nodes $(2,3$, 4, etc. up to 20). We then performed a one-tailed Wilcoxon rank sum test for each group (for 0 versus 1 or 1 versus $2+$ enhancers), and combined the $P$ values by Stouffer's z-score method, to compute two-tailed $P$ values. The $P$ values were also significant $\left(P<10^{-300}\right)$ without this consideration for distance and network degree.

\section{Gene ontology analyses of interconnected gene pairs} We tested if gene pairs connected through promoterpromoter, promoter-enhancer-promoter interactions more often shared annotated gene function. To this end, we used the gene ontology service DAVID [35]. First we calculated for each gene ontology term how many gene pairs were connected through one or more HiCap interactions in the patterns outlined in Fig. $3 \mathrm{~m}$ for genes associated with that gene ontology term. Then we randomized $(\mathrm{n}=1000)$ all HiCap interactions among all promoters and enhancers and repeated the same analyses above. We computed $P$ values as the number of randomizations with at least as many pairs as the nonrandomized, or one less (to account for selecting terms with at least one real pair associated with them). Due to the 1000 randomizations, the minimum possible $P$ value was 0.001 . $P$ values were then adjusted to false discovery rates using the Benjamini-Hochberg method.

\section{Data access}

Raw sequence reads have been submitted to the NCBI Sequence Read Archive [36] under [SRA:SRP045579] and [SRA:SRP045580], and processed gene expression values and interaction files have been submitted to the NCBI GEO [37] under accession number [GEO:GSE60495].

\section{Additional files}

Additional file 1: Supplementary tables. (ZIP $20649 \mathrm{~kb}$ )

Additional file 2: Supplementary figures. (PDF $1269 \mathrm{~kb}$ )

\begin{abstract}
Abbreviations
3C: chromatin conformation capture; 4C: chromosome conformation capture coupled with sequencing; bp: base pair; ChIA-PET: chromatin interaction analysis by paired-end tag sequencing; ChIP-seq: chromatin immunoprecipitation followed by high-throughput DNA sequencing; GEO: Gene Expression Omnibus; mESC: mouse embryonic stem cell; RPKM: reads per kilobase of gene model and million uniquely mapped reads; TAD: topologically associating domain; TF: transcription factor.
\end{abstract}

\section{Competing interests}

P.S., T.J.A. and RS declare competing financial interest: T.J.A. is a stockowner and paid employee at Roche Diagnostics; P.S. and R.S. have applied for a patent relating to the method.

\section{Authors' contributions}

P.S. conceived the method, supervised the HiCap development, generated HiCap libraries, implemented interaction-calling software, analyzed data and contributed text to the manuscript. I.A. developed HiCap, generated HiCap libraries, analyzed data, and contributed text and figures to the manuscript. D.R. made sequence-capture designs, analyzed data, and contributed text and figures to the manuscript. L.M. cultured cells, N.R. performed Capture-C libraries, B.L. performed a subset of sequence captures. T.J.A. generated sequence capture probes. J.L. provided project guidance. R.S. designed the study and prepared the manuscript, with input from the other authors. All authors read and approved the final manuscript.

\section{Acknowledgements}

We thank R. Månsson for constructive comments on the manuscript, and National Genomics Infrastructure and Uppnex for providing access to MPS support and infrastructure. This work was funded by Swedish Foundation for Strategic Research (FFL4, R.S. and J.L.) and Swedish Research Council grants (R.S., J.L.), European Research Council Starting Grant 243066 (R.S.) and Wenner-Gren Foundation fellowship (P.S.).

\section{Author details}

${ }^{1}$ KTH - Royal Institute of Technology, Science for Life Laboratory, School of Biotechnology, Solna 171 65, Sweden. ${ }^{2}$ Ludwig Institute for Cancer Research, Stockholm 171 77, Sweden. ${ }^{3}$ Department of Cell and Molecular Biology, Karolinska Institutet, Stockholm 171 77, Sweden. ${ }^{4}$ Roche Nimblegen Inc., R\&D, Madison, WI 53719, USA.

Received: 1 June 2015 Accepted: 17 July 2015

Published online: 03 August 2015

\section{References}

1. Shlyueva D, Stampfel G, Stark A. Transcriptional enhancers: from properties to genome-wide predictions. Nat Rev Genet. 2014;15:272-86.

2. Arnold CD, Gerlach D, Stelzer C, Boryń ŁM, Rath M, Stark A. Genome-wide quantitative enhancer activity maps identified by STARR-seq. Science. 2013;339:1074-7.

3. Johnson DS, Mortazavi A, Myers RM, Wold B. Genome-wide mapping of in vivo protein-DNA interactions. Science. 2007;316:1497-502.

4. Visel A, Blow MJ, Li Z, Zhang T, Akiyama JA, Holt A, et al. ChIP-seq accurately predicts tissue-specific activity of enhancers. Nature. 2009:457:854-8.

5. Heintzman ND, Hon GC, Hawkins RD, Kheradpour P, Stark A, Harp LF, et al. Histone modifications at human enhancers reflect global cell-type-specific gene expression. Nature. 2009;459:108-12.

6. Boyle AP, Davis S, Shulha HP, Meltzer P, Margulies EH, Weng Z, et al. High-resolution mapping and characterization of open chromatin across the genome. Cell. 2008;132:311-22.

7. Müeller-Storm HP, Sogo JM, Schaffner W. An enhancer stimulates transcription in trans when attached to the promoter via a protein bridge. Cell. 1989;58:767-77.

8. Dekker J, Rippe K, Dekker M, Kleckner N. Capturing chromosome conformation. Science. 2002;295:1306-11.

9. de Wit E, Bouwman BAM, Zhu Y, Klous P, Splinter E, Verstegen MJAM, et al. The pluripotent genome in three dimensions is shaped around pluripotency factors. Nature. 2013;501:227-31.

10. Denholtz M, Bonora G, Chronis C, Splinter E, de Laat W, Ernst J, et al. Long-range chromatin contacts in embryonic stem cells reveal a role for pluripotency factors and polycomb proteins in genome organization. Cell Stem Cell. 2013:13:602-16.

11. Lieberman-Aiden $E$, van Berkum NL, Williams L, Imakaev M, Ragoczy T, Telling $A$, et al. Comprehensive mapping of long-range interactions reveals folding principles of the human genome. Science. 2009;326:289-93.

12. Dixon JR, Selvaraj S, Yue F, Kim A, Li Y, Shen Y, et al. Topological domains in mammalian genomes identified by analysis of chromatin interactions. Nature. 2012;485:376-80.

13. Jin F, Li Y, Dixon JR, Selvaraj S, Ye Z, Lee AY, et al. A high-resolution map of the three-dimensional chromatin interactome in human cells. Nature. 2013;503:290-4.

14. Fullwood MJ, Liu MH, Pan YF, Liu J, Xu H, Mohamed YB, et al. An oestrogen-receptor-alpha-bound human chromatin interactome. Nature. 2009;462:58-64. 
15. Li G, Ruan X, Auerbach RK, Sandhu KS, Zheng M, Wang P, et al. Extensive promoter-centered chromatin interactions provide a topological basis for transcription regulation. Cell. 2012;148:84-98.

16. Hughes JR, Roberts N, McGowan S, Hay D, Giannoulatou E, Lynch M, et al. Analysis of hundreds of cis-regulatory landscapes at high resolution in a single, high-throughput experiment. Nat Genet. 2014;46:205-12.

17. Schoenfelder S, Furlan-Magaril M, Mifsud B, Tavares-Cadete F, Sugar R, Javierre $\mathrm{B}-\mathrm{M}$, et al. The pluripotent regulatory circuitry connecting promoters to their long-range interacting elements. Genome Res. 2015;25:582-97.

18. Mifsud B, Tavares-Cadete F, Young AN, Sugar R, Schoenfelder S, Ferreira L, et al. Mapping long-range promoter contacts in human cells with highresolution capture Hi-C. Nat Genet. 2015;47:598-606.

19. Ma W, Ay F, Lee C, Gulsoy G, Deng X, Cook S, et al. Fine-scale chromatin interaction maps reveal the cis-regulatory landscape of human lincRNA genes. Nat Methods. 2015;12:71-8.

20. Daley T, Smith AD. Predicting the molecular complexity of sequencing libraries. Nat Methods. 2013;10:325-7.

21. HiCUP (Hi-C User Pipeline): A tool for mapping and performing quality control on Hi-C data. http://www.bioinformatics.babraham.ac.uk/projects/hicup/.

22. Zhang Y, Wong C-H, Birnbaum RY, Li G, Favaro R, Ngan CY, et al. Chromatin connectivity maps reveal dynamic promoter-enhancer long-range associations. Nature. 2013;504:306-10.

23. Albert $\mathrm{R}$, Jeong $\mathrm{H}$, Barabasi $A$. Error and attack tolerance of complex networks. Nature. 2000;406:378-82.

24. Osborne CS, Chakalova L, Brown KE, Carter D, Horton A, Debrand E, et al. Active genes dynamically colocalize to shared sites of ongoing transcription. Nat Genet. 2004;36:1065-71.

25. Schoenfelder S, Sexton T, Chakalova L, Cope NF, Horton A, Andrews S, et al. Preferential associations between co-regulated genes reveal a transcriptional interactome in erythroid cells. Nat Genet. 2010;42:53-61.

26. Lizio M, Harshbarger J, Shimoji H, Severin J, Kasukawa T, Sahin S, et al. Gateways to the FANTOM5 promoter level mammalian expression atlas. Genome Biol. 2015;16:22.

27. Dryden NH, Broome LR, Dudbridge F, Johnson N, Orr N, Schoenfelder S, et al. Unbiased analysis of potential targets of breast cancer susceptibility loci by Capture Hi-C. Genome Res. 2014;24:1854-68.

28. Storvall H, Ramsköld D, Sandberg R. Efficient and comprehensive representation of uniqueness for next-generation sequencing by minimum unique length analyses. PLoS One. 2013;8, e53822.

29. Langmead B, Trapnell C, Pop M, Salzberg SL. Ultrafast and memory-efficient alignment of short DNA sequences to the human genome. Genome Biol. 2009;10:R25

30. Wang ET, Sandberg R, Luo S, Khrebtukova I, Zhang L, Mayr C, et al. Alternative isoform regulation in human tissue transcriptomes. Nature. 2008;456:470-6.

31. Ramsköld D, Wang ET, Burge CB, Sandberg R. An abundance of ubiquitously expressed genes revealed by tissue transcriptome sequence data. PLoS Comput Biol. 2009;5, e1000598.

32. Chen $X, X u H$, Yuan P, Fang F, Huss M, Vega VB, et al. Integration of external signaling pathways with the core transcriptional network in embryonic stem cells. Cell. 2008;133:1106-17.

33. Correa-Cerro LS, Piao Y, Sharov AA, Nishiyama A, Cadet JS, Yu H, et al. Generation of mouse ES cell lines engineered for the forced induction of transcription factors. Sci Rep. 2011;1:167

34. Jonkers I, Kwak H, Lis JT. Genome-wide dynamics of Pol II elongation and its interplay with promoter proximal pausing, chromatin, and exons. Elife. 2014;3, e02407. doi:10.7554/eLife.02407.

35. Huang DW, Sherman BT, Lempicki RA. Bioinformatics enrichment tools: paths toward the comprehensive functional analysis of large gene lists. Nucleic Acids Res. 2009;37:1-13.

36. Sequence Read Archive. http://www.ncbi.nlm.nih.gov/sra

37. Gene Expression Omnibus. http://www.ncbi.nlm.nih.gov/geo.

\section{Submit your next manuscript to BioMed Central and take full advantage of:}

- Convenient online submission

- Thorough peer review

- No space constraints or color figure charges

- Immediate publication on acceptance

- Inclusion in PubMed, CAS, Scopus and Google Scholar

- Research which is freely available for redistribution

Submit your manuscript at www.biomedcentral.com/submit 